\title{
Computational prediction and experimental verification of miRNAs in Panicum miliaceum L.
}

\author{
WU YongJun ${ }^{1}$, DU JiangFeng ${ }^{1}$, WANG XiaoLong ${ }^{1}$, FANG XiaoFeng ${ }^{3}$, SHAN WeiXing ${ }^{2}$ \& \\ LIANG ZongSuo ${ }^{1 *}$
}

${ }^{1}$ College of Life Sciences, Northwest Agriculture \& Forestry University, Yangling 712100, China;
${ }^{2}$ College of Plant Protection, Northwest Agriculture \& Forestry University, Yangling 712100, China;

${ }^{3}$ National Institute of Biological Sciences, Beijing 102206, China

Received January 2, 2012; accepted July 12, 2012

\begin{abstract}
MicroRNAs (miRNAs) are a class of non-coding RNAs that play critical roles in post-transcriptional regulation. Their target genes are involved in a variety of biological processes such as development, metabolism, and stress response. Panicum miliaceum L. (Panicum) is an important grain crop, but, until now, no miRNAs have been identified in this plant. Using a homology search based on expressed sequence tag (EST) analysis and miRNA precursor secondary structure, a total of 43 new miRNAs were identified. The miRNAs were found to be unevenly distributed among 11 miRNA families. Target analysis using the plant small RNA target analysis server psRNATarget showed that the newly identified miRNAs can potentially regulate 68 target genes. Ten of the 11 miRNA families were annotated as involved in RNA regulation, suggesting they may play an essential role in post-transcriptional regulation in Panicum. Selected miRNAs representing eight of the families were verified by northern blotting, indicating that the prediction method that we used to identify the miRNAs was effective.
\end{abstract}

microRNA, Panicum, prediction, verification, targets

Citation: Wu Y J, Du J F, Wang X L, et al. Computational prediction and experimental verification of miRNAs in Panicum miliaceum L.. Sci China Life Sci, 2012, 55: 807-817, doi: 10.1007/s11427-012-4367-y

MicroRNAs (miRNAs) are non-coding RNA molecules with important regulatory functions in gene expression. They originate from long self-complementary (stem-loop) precursors (pre-miRNA) consisting of 60-160 nucleotides (nt). The majority of known mature miRNAs are about 21$25 \mathrm{nt}$ long and several steps are involved in their processing and maturation [1,2]. MiRNAs are widely distributed in plant, animal and even virus genomes and most of them have independent genetic loci and show conservation between different species of the same kingdom [3]. MiRNAs can direct the RISC (RNA-induced silencing complex) to post-transcriptionally regulate gene expression through messenger RNA (mRNA) decomposition or translational

*Corresponding author (email: Liangzs@ms.iswc.ac.cn) repression [4]. The target sites on the mRNA that show full complementarity with a miRNA can be specifically degraded; if there are mismatches, the mRNA translation can be inhibited.

MiRNA-regulated genes control a variety of biological and metabolic processes. For example, in animals, miRNAs regulate developmental timing, stem cell maintenance and differentiation, organ development, signal transduction, disease, and cancer pathogenesis [5-7]; in plants, miRNAs regulate leaf, stem, root, and flower development, phase switch from vegetative growth to reproductive growth, and responses to abiotic and biotic stress [8]. Several studies have indicated that miRNAs directly target the transcription factors that regulate plant development as well as specific genes that control various metabolic processes [9-11]. 
A total of 16772 miRNAs were recorded in the April 2011 release of the miRBase database (http://www.mirbase. org/). The entries included 411 and 491 miRNAs in Arabidopsis thaliana and Oryza sativa, respectively. Until now, no miRNA has been reported in Panicum miliaceum L., a popular grain crop around the world, which has many advantageous attributes such as a short reproductive cycle, drought resistance, and leanness-tolerance [12]. Panicum not only serves as a minor cereal in food supply providing humans with a balanced diet, but also is a source of beer, yellow wine and beverage production [13]. The identification of miRNAs in Panicum may provide new insights into understanding the mechanisms of the many biological processes that are involved in its cultivation and growth of this important plant.

Three strategies are available currently to identify miRNAs: direct cloning, forward genetics and bioinformatics, and a large number of miRNAs have been identified using these strategies. Direct cloning is the most effective and reliable method, through which the first miRNAs in plants were identified [14]. However, some technical difficulties make direct cloning and sequencing of short RNA molecular hard to implement [15]. For example, miRNAs tend to have highly constrained tissue and time-specific expression patterns; in addition, degradation products of mRNAs and other endogenous non-coding RNAs co-exist with miRNAs and these are sometimes dominant in sample containing small RNAs. These factors make it difficult to validate miRNAs using experiment approaches. Because miRNAs are conserved between different species and pre-miRNAs have a unique secondary structure [3,16-20], these characteristics have been used for miRNA prediction using bioinformatics [21]. Many novel miRNAs have been identified through this kind of strategy $[6,9,15,22-26]$. Jones-Rhoades and Bartel [9] developed a comparative genomic approach to systematically identify both miRNAs and their targets. Zhang and co-workers [24] identified 338 new candidate miRNAs in 60 plants by scanning a total of 18694 plant expressed sequence tags (ESTs) [27-29]. Later, the same workers [6] reported 30 potential cotton miRNAs based on genomic survey sequence analysis and miRNA secondary structure. Although most recent research has relied on genomic survey sequence analysis to predict miRNAs, predictions based on ESTs can still produce significant results. In this study, we compared Panicum EST sequences with all the previously known miRNAs in the miRBase database to identify candidate miRNAs in Panicum. To help us understand the biological processes in which they might be involved, we also predicted the potential targets of the candidate miRNAs [30]. To prove their validity, some of the computationally predicted miRNAs were verified by northern blotting hybridization, an effective and widely-used method for detecting miRNAs.

\section{Materials and methods}

\section{1 miRNA and EST sequences datasets}

A total of 8273 reported mature miRNAs and their pre-miRNA sequences were obtained from the September 2008 miRBase database (ftp://mirbase.org/pub/mirbase/ 12.0/). Because some miRNAs show conservation across different kingdoms [31], we included the miRNAs not only from plants such as Arabidopsis thaliana, Zea mays, Oryza sativa, Glycine max, and Sorghum bicolor, but also from animals like Homo sapiens, Mus musculus, Caenorhabditis elegans, and Drosophila melanogaster. To avoid overlap of the miRNAs, any repeated sequences were removed and the remaining sequences were used as the reference miRNAs dataset. A total of 436736 Panicum sequences were downloaded from the 2008 release of the NCBI (National Center for Biotechnology Information) GenBank EST databases (http://www.ncbi.nlm.nih.gov/).

\subsection{Identification of candidate miRNAs in the Panicum EST dataset}

BLAST (basic local alignment search tool) [32] was used to search all the reported mature miRNA sequences in the dataset against the downloaded EST sequences. The BLAST parameters were essentially the same as described previously $[6,24,26,33,34]$; the expected value was set to 1000 and the minimum word size was 7. A stable version of the BLAST tool was downloaded from http://blast.ncbi.nlm. nih.gov/Blast.cgi. Two rules [24,26,33] were adopted during the alignment process: first, less than three mismatches were allowed; and second, the percent identity should be no less than $(L-3+M) / L$, where $L$ is the length of the Hits (see Bio::SearchIO (Dr. Jason Stajich) http://www.bioperl.org/ wiki/HOWTO:SearchIO\#Abstract), and $M$ is the number of mismatches. A total of 6079 ESTs that potentially contain miRNA sequences were obtained after the BLAST search (Figure 1).

\subsection{Identification of pre-miRNA sequences}

Plant pre-miRNAs are usually about $70 \mathrm{nt}$ long, while the stem-loop structures of plant pri-miRNAs vary greatly in length (from 100 to $1000 \mathrm{nt}$ ) [35]. In this study, we chose 60-250 nt as the length of potential precursors to obtain more precursors and used the RNAShapes software package (http://bibiserv.techfak.uni-bielefeld.de/rnashapes/) [36-38] to compute their structure. We moved a sliding segment of 60-250 nt long along each of the selected EST sequences and extracted any segment that had the same length as the sliding segment. Five base pairs were selected as the scanning window and three base pairs as the stretching window during extraction. The RNAShapes parameters [39] were set as follows: shape probabilities selected, and probability 


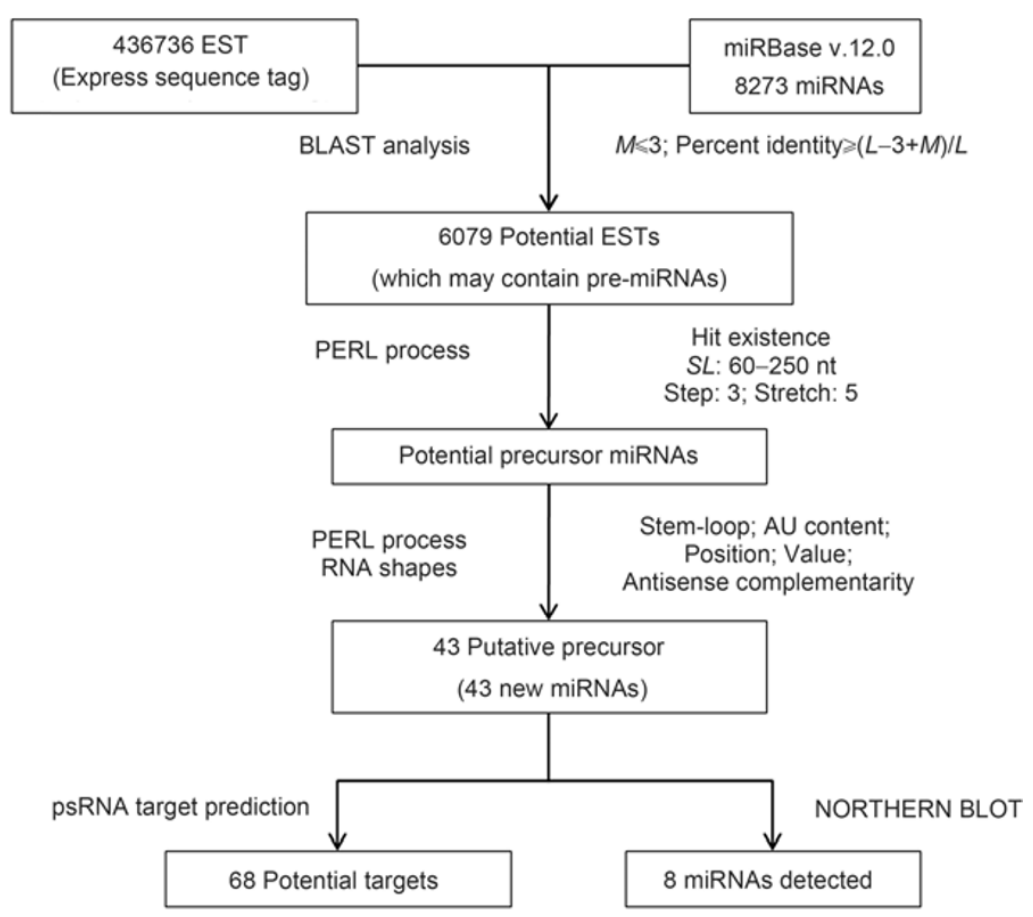

Figure 1 Flowchart for the prediction and verification of the Panicum miRNAs. $M$, mismatch number; $L$, length of hits; $S L$, length of the sliding segment; Position, position of the hit in the sliding segment; Value, the absolute value of the difference between the length of mature miRNA and the number of matches.

output filter as 0.5 . The following criteria [24,26,40] were adopted to screen potential precursors further: (i) the threshold free energy for the precursor sequences was set to $-25 \mathrm{kcal} \mathrm{mol}^{-1}$; (ii) the $(\mathrm{A}+\mathrm{U})$ content should be between $52.0 \%$ and $68.0 \%$; (iii) the mature miRNA sequence should be on the stem of the precursor sequence; and (iv) the value (the absolute value of the difference between the length of mature miRNA and the number of matches) was less than 5 (Figure 1).

\subsection{Prediction of the potential targets}

We predicted the potential targets of the newly identified candidate miRNAs using the psRNATarget server at http://www.plantgrn.org/psRNATarget/ [40]. All the parameters [40] except the dataset employed were set to the default. Because genome information of Panicum is not available, the dataset was set first as the TIGR Gene Index (http://compbio.dfci.harvard.edu/tgi/tgipage.html) of the species from which the original miRNA sequence came, then as the Arabidopsis Gene Index, and then as the Rice Gene Index (Figure 1).

\subsection{Verification of the predicted miRNAs}

Two cultivars of Panicum, Longmi-5 and Ningmi-9 were planted and harvested after $20 \mathrm{~d}$. Whole-plant total RNA was extracted using Trizol reagent (Tiangen, Beijing, China) following the manufacturer's instruction. The procedure of northern blot was based on a published article [41] with minor modifications. Because of the high sequence similarity of miRNA members within a family, we chose $12 \mathrm{miR}$ NAs that represented 11 families for verification (Table 1).

\section{Results and discussion}

\subsection{Prediction of potential miRNAs}

In the plant kingdom, most mature miRNAs are highly conserved across different species [8], and this characteristic makes it possible to predict novel miRNAs based on sequence alignments. The BLAST searches of known miRNAs against the Panicum ESTs dataset identified 6079 potential miRNAs-containing ESTs. Further screening of their secondary structures, free energy, and base content, identified a total of 43 potential mature miRNAs in the $P$. miliaceum L. dataset (Table 2). The candidate miRNAs were distributed unevenly among 11 miRNA families. Ten of the miRNAs were members of the miR159 family, and eight were members of the miR169 family, while only one miR171 and one miR1439 were found (Figure 2). This distribution suggested that the Panicum miRNAs were as diverse as the miRNAs from A. thaliana, Z. mays and $O$. sativa (Table 3).

Many miRNA genes are found in multiple locations and the candidate miRNAs that we found were often found in different ESTs with unique gene numbers. Thirteen of the 43 identified miRNAs corresponded to more than one gene number. For example, miR159b-6 matched ESTs with three different gene members, miR444b-1 matched four, and 
Table 1 Probes used in the Northern blot assay

\begin{tabular}{lll}
\hline \multicolumn{1}{c}{ Probe } & \multicolumn{1}{c}{ Sequence } & Targets \\
\hline $\mathrm{R}$ & TACGATCAGCATTGCTAGCGA & 5S rRNA degradation product \\
EST1 & CTGGTAGTAGTGTAGATTGCT & Random EST degradation product \\
EST2 & CATGAAGACCACATAAGGAGC & $\mathrm{miR} 156 \mathrm{a} 1 / 2 / 3$ \\
156 & TGTGCTCACTCTCTTCTGTCA & $\mathrm{miR} 159 \mathrm{a}$ \\
159 & GGGAGCACCCTTCAGTCCAA & $\mathrm{miR} 160-1 / 2$ \\
160 & TGGCATACAGGGAGCCAGGCA & $\mathrm{miR} 166 \mathrm{a} / \mathrm{b}$ \\
166 & AGGGGAATGAAGCCTGGTCCGA & $\mathrm{miR} 167 \mathrm{a} / \mathrm{b}-1 / 2$ \\
167 & TAGATCATGCTGGCAGCTTCA & $\mathrm{miR} 169 \mathrm{~d}$ \\
169 & TAAGCAAGTCATCCTTGGCTA & $\mathrm{miR} 171$ \\
171 & TAAGATATTGGCACGGCTCAA & $\mathrm{miR} 399 \mathrm{~b}$ \\
499 & CCGGGCAAATCTCCTTTGGCA & $\mathrm{miR} 444 \mathrm{a}-1 / 2 / 3$ \\
$444 \mathrm{a}$ & AAGCTTGAGGCAACAACTGCA & $\mathrm{miR} 444 \mathrm{~b}-1 / 2 / 3$ \\
528 & GGCAGCAAGCTTGAGACAACA & $\mathrm{miR} 528 \mathrm{~b}$ \\
1439 & CCCCTCTGCATGCCCCTTCCA & $\mathrm{miR} 1439$ \\
\hline
\end{tabular}

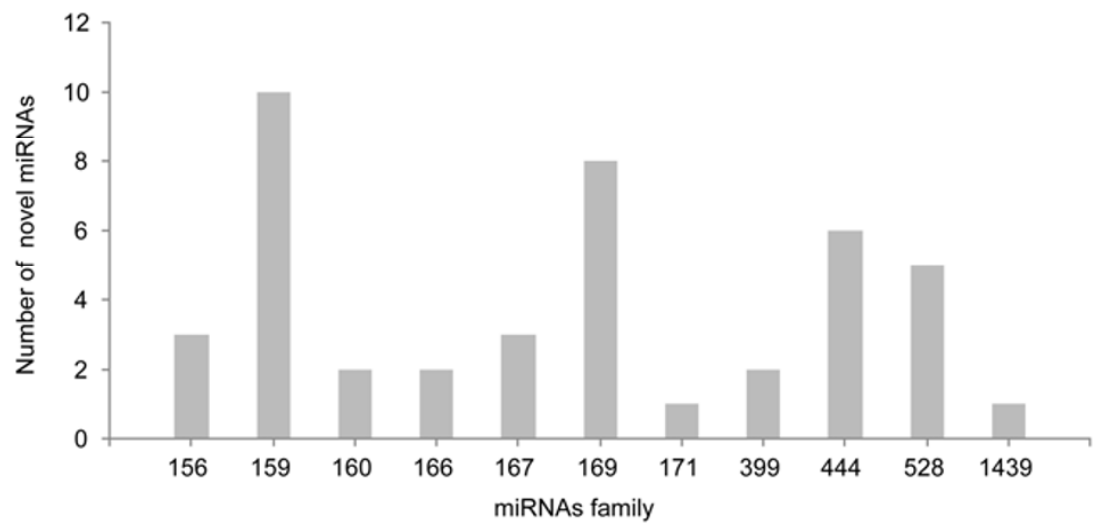

Figure 2 Number of new Panicum candidate miRNAs.

miR528a-2 matched six (Table 2, Figure 2). One possible reason for this is that very few studies have been done on the Panicum genome [42] and some of the ESTs with distinct gene numbers (Table 2) may turn out to be identical when better assemblies of the genome become available. The apparent differences in the EST sequences may result either from mistakes, repeats in sequencing, or discrepancies between the different groups that generated the sequence [43-47]. Another possibility is that the ESTs correspond to the same miRNA are copies of each other and are expressed under difference circumstances: (i) the copies are functionally complementary to each other, and malfunction of one of the copies is complemented by another; (ii) their expression is tissue- and/or time-specified; and (iii) they are expressed simultaneously.

In contrast to most miRNA precursors in other plants, the precursors in Panicum show great diversity. Although the lengths of the miRNA precursors ranged from 84 to $273 \mathrm{nt}$ with an average of $161 \mathrm{nt}$, approximately $50 \%$ of them were 84-126 nt long (Figure 3). The different sizes of the candidate miRNAs within different families suggested that the different precursors might offer unique functions for the regulation of miRNA biogenesis or gene regulation [33]. Their minimal free energy (MFE) ranged from 43.20 to $118.6 \mathrm{kcal}$ and their minimal free energy index (MFEI) ranged from 0.580 to 0.884 , which is acceptable. Mature miRNAs are usually located on each arm of the stem-loop hairpin structure; some at the $5^{\prime}$ end of the miRNA precursor sequences, and others at the $3^{\prime}$ end. Among the 43 newly identified Panicum miRNAs, 22 were located at the $3^{\prime}$ end and 21 were at the $5^{\prime}$ end (Table 2).

Although all the miRNAs from all the species in the miRBase database were included in our dataset, it turned out that the candidate Panicum miRNAs that we identified were only those that were conserved in other plants including Oryza sativa, Triticum aestivum, Sorghum bicolor, Selaginella moellendorffii, Solanum lycopersicum, Brassica napus, Vitis vinifera, Populus trichocarpa and Arabidopsis thaliana (Table 3). Indeed, 36 of 43 candidate Panicum miRNAs showed full complementarity with their counterparts in other plants; the other seven miRNAs had mismatches, especially in the $3^{\prime}$ end. For example, three mismatches were found for miR159a, miR171, and miR1439; and two were found for miR169c and miR169d (Table 2). 
Table 2 Panicum miRNAs identified by homolog search and secondary structure ${ }^{\text {a) }}$

\begin{tabular}{|c|c|c|c|c|c|c|c|c|c|c|c|c|}
\hline miRNA & Iature sequences & PL & Gene number & Loc & ML & $\mathrm{A} \%$ & $\mathrm{C} \%$ & $\mathrm{U} \%$ & $\mathrm{G} \%$ & $(\mathrm{~A}+\mathrm{U}) \%$ & MFE & MFEI \\
\hline $156 a-1$ & GACAGAAGAGAGUGAGCACA & 98 & gi198367980, gi1 & $5^{\prime}$ & 21 & 0.235 & 0.245 & 0.265 & 0.255 & 0.500 & 55.20 & 0.767 \\
\hline $156 a-2$ & GACAGAAGAGAGUGAGCACA & 84 & 601 & $5^{\prime}$ & 21 & 262 & 0.250 & .262 & 0.226 & .524 & 44.80 & .723 \\
\hline $156 a-3$ & GAGAGUGAGCACA & 84 & 198186083, g & $5^{\prime}$ & 21 & 0.214 & 0.214 & 0.274 & 0.298 & 488 & 3.90 & .884 \\
\hline $159 \mathrm{a}$ & $\mathrm{CC}$ & 174 & 46 & $3^{\prime}$ & 20 & 218 & 0.236 & 0.276 & 0.270 & 0.494 & 91.30 & .725 \\
\hline $159 b-1$ & UUUGGAUUGAAGGGAGCUCUG & 257 & 198383357, g & $3^{\prime}$ & 21 & 0.175 & 0.226 & 0.292 & 0.307 & 0.467 & 105.47 & 0.580 \\
\hline $159 \mathrm{~b}-2$ & UUUC & 254 & 8350925 & $3^{\prime}$ & 21 & & 0.232 & 0.287 & & & & 629 \\
\hline $159 b-3$ & UUU & 248 & 3 & $3^{\prime}$ & 21 & 0.177 & 0.226 & 0.286 & 0.310 & 0.464 & 111.17 & 628 \\
\hline $159 b-4$ & JGAAGGGAGCUCUG & 259 & 85 & $3^{\prime}$ & 21 & 0.178 & 0.228 & 0.286 & 0.309 & 0.463 & 116.12 & 0.628 \\
\hline $159 \mathrm{~b}-5$ & UUUGGAUUGAAGGGAGCUCUG & 267 & gil9 & $3^{\prime}$ & 21 & 0.172 & 0.236 & 0.288 & 0.303 & 61 & 118.82 & 0.625 \\
\hline $159 \mathrm{~b}-6$ & $\mathrm{U}$ & 259 & $\begin{array}{l}\text { gi198068022, gi197980325 } \\
\text { gi169675227 }\end{array}$ & $3^{\prime}$ & 21 & 74 & 0.228 & 0.290 & 0.309 & 0. & 22 & 615 \\
\hline $159 b-7$ & UUUGGAUUGAAGGGAGCUCUG & 273 & -0.1002060าร & $3^{\prime}$ & 21 & 0.168 & 0.231 & 0.300 & 0.300 & 469 & 60 & 0.621 \\
\hline $159 b-8$ & UUUGGAUUGAAGGGAGCUCUG & 263 & g1190 & $3^{\prime}$ & 21 & 75 & 0.236 & 0.297 & 0.293 & 71 & & 0.600 \\
\hline $159 b-9$ & UUL & 242 & & $3^{\prime}$ & 21 & 0.165 & 0.227 & 0.293 & 0.314 & & & 643 \\
\hline $160-1$ & UGCC & 133 & gi19 & $5^{\prime}$ & 21 & 0.195 & 0.278 & 0.203 & 0.323 & 0.398 & 00 & 0.623 \\
\hline $160-2$ & UGCC & 128 & $\mathrm{~g}$ & $5^{\prime}$ & 2 & & 0.313 & 0.219 & 0.313 & & & 714 \\
\hline $166 \mathrm{a}$ & $\mathrm{CU}$ & 117 & & $3^{\prime}$ & 22 & 0.171 & 0.222 & 0.333 & 0.274 & & & 855 \\
\hline $166 \mathrm{~b}$ & UCGG & 189 & gi19830 & $3^{\prime}$ & 22 & 0.148 & 0.275 & 0.275 & 0.302 & 0.423 & & 0.738 \\
\hline $167 a-1$ & UGA & 106 & gi198 & $5^{\prime}$ & 21 & & 0.255 & 0.236 & & & & .770 \\
\hline $167 a-2$ & JA & 118 & & $5^{\prime}$ & 21 & 0.212 & 0.271 & 0.229 & 0.288 & 41 & 10 & .781 \\
\hline $167 \mathrm{~b}$ & UGA & 155 & & $5^{\prime}$ & 21 & 0.219 & 0.200 & 0.290 & 0.290 & & & 0.703 \\
\hline $169 a-1$ & UAG & 209 & & $5^{\prime}$ & 21 & & 0.234 & 0.282 & 0.297 & 69 & & 0.663 \\
\hline $169 a-2$ & UAC & 208 & & $5^{\prime}$ & 21 & 0.183 & 0.274 & 0.269 & 0.274 & 51 & .70 & .709 \\
\hline $169 a-3$ & UAGC & 202 & oil19: & $5^{\prime}$ & 21 & 0.158 & 0.262 & 0.277 & 0.302 & 0.436 & & 0.771 \\
\hline $169 a-4$ & UAG & 200 & & $5^{\prime}$ & 21 & 0.160 & 0.270 & 0.260 & 0.310 & 0 & & 0.751 \\
\hline $169 b-1$ & UAC & 175 & & $5^{\prime}$ & 21 & 0.229 & 0.223 & 0.286 & 0.263 & 14 & 30 & 0.730 \\
\hline $169 b-2$ & $\mathrm{UA}$ & 172 & & $5^{\prime}$ & 21 & 0.233 & 0.233 & 0.285 & 0.250 & & & 0.696 \\
\hline $169 \mathrm{c}$ & UAG & 111 & & $5^{\prime}$ & 20 & & 0.243 & 0.279 & 0.324 & & & 0.706 \\
\hline $169 \mathrm{~d}$ & UAC & 111 & & $5^{\prime}$ & 21 & 0.153 & 0.243 & 0.279 & 0.324 & 0.432 & 40 & 0.705 \\
\hline 171 & UUGAGCCGUGCCAAUAUCUUA & 117 & gi198 & $3^{\prime}$ & 21 & 0.205 & 0.222 & 0.308 & 0.265 & 0.513 & 51.70 & 0.638 \\
\hline $399 \mathrm{a}$ & UGCCAAAGGAGAUUUGCCCGG & 104 & & $3^{\prime}$ & 21 & & 0.337 & 0.163 & 0.317 & & & 0.744 \\
\hline $399 \mathrm{~b}$ & UGC & 166 & $\mathrm{~g}$ & $3^{\prime}$ & 21 & 0.235 & 0.205 & 0.319 & 0.241 & 0.554 & 00 & 0.575 \\
\hline $444 a-1$ & UGCAGUUGUUGCCUCAAGCUU & 123 & & $3^{\prime}$ & 21 & 0.276 & 0.228 & 0.260 & 0.236 & 0.537 & 72.11 & 0.792 \\
\hline $444 a-2$ & & 123 & & $3^{\prime}$ & 21 & & 0.228 & 0.260 & 0.236 & & & 0.792 \\
\hline $444 a-3$ & UGC & 116 & 0 & $3^{\prime}$ & 21 & 0.267 & 0.216 & 0.267 & 0.250 & 0.534 & 70.90 & 0.834 \\
\hline 444b-1 & $\mathrm{U}$ & 12 & $\begin{array}{l}\text { gi } 19 \\
\text { gi } 19\end{array}$ & $3^{\prime}$ & 2 & 2 & 2 & 02 & 0.254 & 0.524 & 74.30 & 0.844 \\
\hline $444 b-2$ & & 126 & gi198 & $3^{\prime}$ & 21 & 0.230 & 0.222 & 0.302 & 0.246 & 0.532 & 70.40 & 0.800 \\
\hline $444 b-3$ & UGUUGUCUCAAGCUUGCUGCC & 126 & gi59871869 & $3^{\prime}$ & 21 & 0.230 & 0.230 & 0.302 & 0.238 & 0.532 & 72.20 & 0.820 \\
\hline $528 \mathrm{a}-1$ & UGGAAGGGGCAUGCAGAGGAG & 90 & & $5^{\prime}$ & 21 & 0.156 & 0.256 & 0.256 & 0.333 & 0.411 & 43.50 & 0.649 \\
\hline 5 & $\mathrm{U}$ & 90 & 336818 & $5^{\prime}$ & 21 & 56 & 0.267 & 44 & 0.333 & 0 & 4 & 76 \\
\hline-3 & $A G$ & 88 & & $3^{\prime}$ & 21 & 0.159 & 73 & 0.239 & 0.330 & 0.3 & 90 & 0.670 \\
\hline 528 & UGGAAGGGGCAL & 90 & $\begin{array}{l}\text { gi198326853, gi } \\
\text { gi198299290, gi }\end{array}$ & $5^{\prime}$ & 21 & 0.156 & 0.267 & 0.256 & 0.322 & 0.411 & 44.60 & 0.666 \\
\hline $528 \mathrm{~b}$ & & 90 & gi198. & $5^{\prime}$ & 21 & & 0.256 & 0.256 & 0.344 & 0.400 & & 0.645 \\
\hline 1439 & AUUUGGAACGGAGUGAGUACA & 263 & gi198019034 & $3^{\prime}$ & 21 & 0.350 & 0.114 & 0.422 & 0.114 & 0.772 & 105.02 & 0.691 \\
\hline
\end{tabular}

a) PL, Length of precursor sequence; Loc, location of mature miRNA in the precursor; ML, length of mature sequence; MFE, minimum free energy; MFEI, minimum free energy index. The shaded letters indicate nucleotide mismatches compared with the corresponding sequences in miRBase.

Because miRNAs are believed to occur at a frequency of approximately $0.1 \%$ of the total EST sequences of a species [33], the 436736 Panicum ESTs in the dataset should contain 43.67 miRNAs. This number is very close to our results, suggesting that the parameters we chose in the prediction process are reasonable and the methods we employed are proper, and, more importantly, the prediction of miRNAs based on ESTs was effective.

\subsection{Prediction and analysis of Panicum miRNA targets}

In this study, we identified a total of 68 potential targets for 
Table 3 Total number of miRNAs and their numbers in the corresponding families in Panicum and some other plant species

\begin{tabular}{|c|c|c|c|c|c|c|c|c|c|c|c|c|c|}
\hline \multirow{2}{*}{ Species } & \multirow{2}{*}{ Total number } & \multirow{2}{*}{ Family } & \multicolumn{11}{|c|}{ The 11 miRNAs families identified in Panicum and the number of miRNAs in each family } \\
\hline & & & 156 & 159 & 160 & 166 & 167 & 169 & 171 & 399 & 444 & 528 & 1439 \\
\hline P. milaceum $\mathrm{L}$. & 43 & 11 & 3 & 10 & 2 & 2 & 3 & 8 & 1 & 2 & 6 & 5 & 1 \\
\hline Oryza sativa & 491 & 213 & 12 & 6 & 6 & 14 & 10 & 17 & 9 & 11 & 6 & 1 & 1 \\
\hline Triticum aestivum & 32 & 31 & 0 & 2 & 1 & 0 & 1 & 0 & 1 & 1 & 1 & 0 & 0 \\
\hline Sorghum bicolor & 148 & 27 & 9 & 2 & 6 & 11 & 9 & 17 & 11 & 11 & 0 & 1 & 0 \\
\hline Zea Mays & 170 & 28 & 12 & 11 & 7 & 14 & 10 & 18 & 14 & 10 & 0 & 2 & 0 \\
\hline Arabidopsis thaliana & 328 & 251 & 16 & 6 & 6 & 14 & 10 & 29 & 7 & 15 & 0 & 0 & 0 \\
\hline Selaginella moellendorffii & 58 & 45 & 4 & 1 & 2 & 3 & 0 & 0 & 4 & 0 & 0 & 0 & 0 \\
\hline Solanum lycopersicum & 36 & 23 & 3 & 1 & 1 & 2 & 1 & 4 & 4 & 1 & 0 & 0 & 0 \\
\hline Brassica napus & 46 & 17 & 3 & 1 & 0 & 4 & 3 & 13 & 7 & 1 & 0 & 0 & 0 \\
\hline Vitis vinifera & 163 & 49 & 9 & 3 & 5 & 8 & 5 & 25 & 9 & 9 & 0 & 0 & 0 \\
\hline Populus trichocarpa & 233 & 42 & 10 & 6 & 8 & 17 & 8 & 32 & 14 & 12 & 0 & 0 & 0 \\
\hline
\end{tabular}

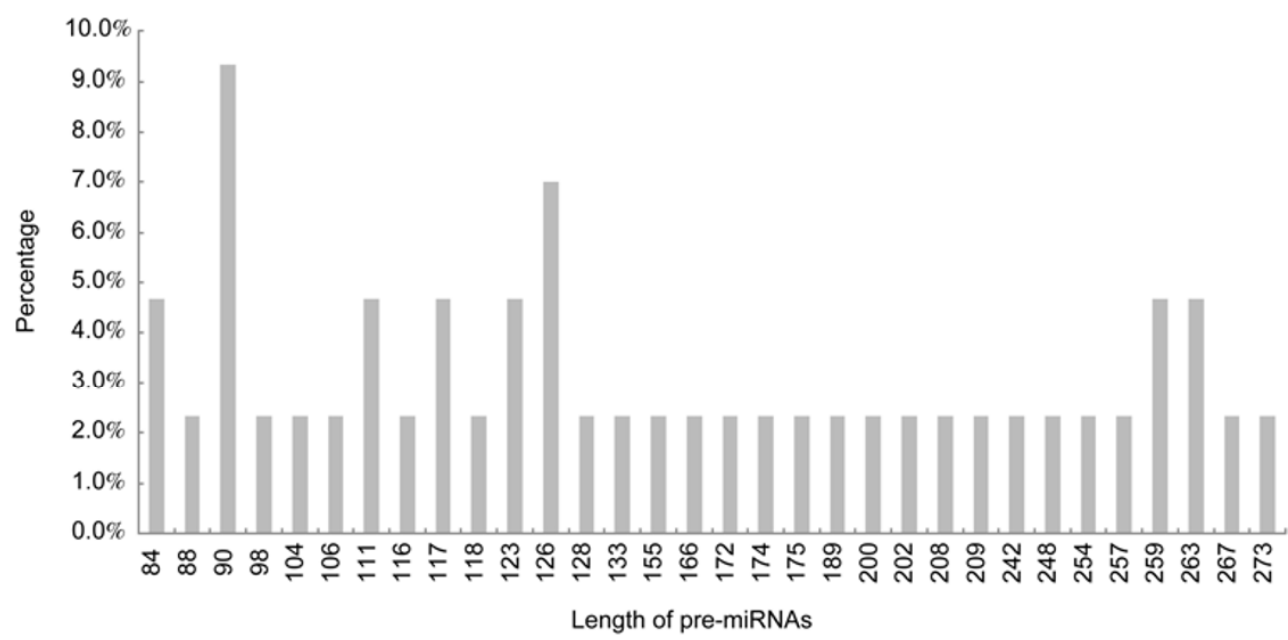

Figure 3 Length distribution of the predicted precursor miRNAs.

the 11 identified miRNA families in Panicum based on the fact that miRNAs show perfect or near-perfect complementary to their target mRNA sequences (Table 4). The 68 potential miRNA targets belong to several gene families and have different biological functions that include RNA regulation, metabolism, stress response, RNA processing, signal transduction and transportation.

It is generally accepted that almost all miRNA targets have no more than four mismatches with their corresponding miRNAs in plants [48], and this criterion has been widely adopted to identify miRNA targets in different plant species. In our study, we allowed no more than three mismatches between miRNAs and their corresponding targets and included no more than one mismatch between 2 and 8 nt of the mature miRNAs as a standard.

Among the 11 identified miRNA families, some are known to be involved in multiple functions (for example, miR166, miR399, and miR1439) while others have unique functions (for example, miR171, miR156, and miR160). All the identified families except miR528 are associated with RNA regulation. For example, miR156 targets the Squamo- sa-promoter binding protein which is a trans-acting factor that binds specifically to the consensus nucleotide sequence 5'-TNCGTACAA-3' [49,50]; and miR159 targets a MYB transcription factor which is a transcription activator [51]. When associated with the BHLH12/MYC1, EGL3, or GL3 transcription factor, MYB protein can promote the synthesis of phenylpropanoid-derived compounds such as anthocyanins and proanthocyanidin [52]. miR160 targets auxin response factors, which are a group of transcriptional factors that bind specifically to the DNA sequence 5'-TGTCTC-3' found in the auxin-responsive promoter elements (AuxREs) [53]. Formation of heterodimers with Aux/IAA proteins may alter their ability to modulate early auxin response genes expression [54]. miR166 targets the homeobox-leucine zipper protein which is a probable transcription factor involved in the regulation of meristem development to promote lateral organ formation and may regulate procambial and vascular tissue formation or maintenance, and vascular development in inflorescence stems [53-55]. Besides these, miR171 targets the F6N15.20 protein, zinc finger (CCCH-type) protein-like, and the Scl1 protein, miR444 
Table 4 Predicted targets of the candidate miRNAs identified in Panicum

\begin{tabular}{|c|c|c|c|}
\hline miRNA & Target protein & Target function & Target gene \\
\hline \multirow[t]{6}{*}{$156 \mathrm{a}$} & Squamosa promoter-binding-like protein & Transcription regulation & $\begin{array}{lllll}\text { TC7137 } & \text { TC28500 } & \text { TC24952 } & \text { TC13039 } & \text { TC36542 } \\
\text { TC19607 } & \text { TC15006 } & \text { TC42015 } & & \end{array}$ \\
\hline & Teosinte glume architecture & Structural protein & TC52769 \\
\hline & SBP transcription factor & Transcription regulation & TC168 ТC17992 ТC41889 \\
\hline & Diacylglycerol kinase-like & Signal transduction & TC50996 \\
\hline & Methyladenine glycosylase protein-like & DNA processing & TC2901 \\
\hline & $\begin{array}{l}\text { Chromosome chr12 scaffold_78, whole genome } \\
\text { shotgun sequence }\end{array}$ & & TC34681 \\
\hline \multirow[t]{2}{*}{$159 \mathrm{a}$} & Transcription factor GAMYB & Transcription regulation & TC4716 \\
\hline & Inositol 1,3,4-trisphosphate 5/6-kinase & Signal transduction & TC1184 \\
\hline \multirow[t]{2}{*}{$159 \mathrm{~b}$} & Transcription factor GAMYB & Transcription regulation & TC4716 \\
\hline & Inositol 1,3,4-trisphosphate 5/6-kinase & Signal transduction & TC1184 \\
\hline 160 & Auxin response factor & Signal transduction & TC52413 ТC20541 ТC3798 \\
\hline \multirow[t]{3}{*}{$166 \mathrm{a}$} & Class III HD-Zip protein & Transcription regulation & TC25111 \\
\hline & $\begin{array}{l}\text { Chromosome chr9 scaffold_7, whole genome } \\
\text { shotgun sequence }\end{array}$ & & FL705256 \\
\hline & MJ0042 family finger-like protein & Transcription regulation & FL805767 \\
\hline \multirow[t]{4}{*}{$166 b$} & Class III HD-Zip protein & Transcription regulation & TC25111 \\
\hline & Rolled leaf & Unknown & TC40382 \\
\hline & $\begin{array}{l}\text { Chromosome chr9 scaffold_7, whole genome } \\
\text { shotgun sequence }\end{array}$ & & FL705256 \\
\hline & MJ0042 family finger-like protein & Transcription regulation & FL805767 \\
\hline $167 \mathrm{a}$ & $\begin{array}{l}\text { Chromosome chr8 scaffold_68, whole genome } \\
\text { shotgun sequence }\end{array}$ & & 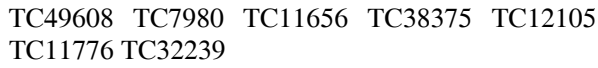 \\
\hline \multirow[t]{3}{*}{$167 b$} & Os05g0109600 protein & Transcription regulation & TC23301 \\
\hline & Expressed protein & Unknown & TC39870 \\
\hline & $\begin{array}{l}\text { Type I secretion outer membrane protein, TolC } \\
\text { family precursor }\end{array}$ & Signal transduction & FL707871 \\
\hline \multirow[t]{4}{*}{$169 \mathrm{a}$} & $\begin{array}{l}\text { CCAAT-binding transcription factor subunit B } \\
\text { family protein }\end{array}$ & Transcription regulation & TC32992 TC41994 ТC33317 ТC50964 ТC5643 \\
\hline & HAP2 subunit of HAP complex & Structural protein & TC6534 TC13896 \\
\hline & $\begin{array}{l}\text { CCAAT-box transcription factor complex } \\
\text { WHAP6 }\end{array}$ & Transcription regulation & TC3877 \\
\hline & Os02g0776400 protein & Transcription regulation & TC33524 \\
\hline \multirow[t]{3}{*}{$169 b$} & $\begin{array}{l}\text { CCAAT-binding transcription factor subunit B } \\
\text { family protein }\end{array}$ & Transcription regulation & TC32992 TC41994 TC33317 TC50964 TC5643 \\
\hline & Os02g0776400 protein & Transcription regulation & TC17857 ТC48466 ТC31225 \\
\hline & XAP5 family protein & & TC32011 \\
\hline \multirow[t]{3}{*}{$169 \mathrm{c}$} & $\begin{array}{l}\text { CCAAT-binding transcription factor subunit B } \\
\text { family protein }\end{array}$ & Transcription regulation & TC32992 TC41994 TC33317 ТC50964 TC5643 \\
\hline & Os05g0363500 protein & Transcription regulation & FL727055 \\
\hline & Os02g0776400 protein & Transcription regulation & TC17857 ТC48466 ТC31225 \\
\hline \multirow[t]{7}{*}{$169 d$} & $\begin{array}{l}\text { CCAAT-binding transcription factor subunit B } \\
\text { family protein }\end{array}$ & Transcription regulation & TC32992 TC41994 TC33317 ТC50964 ТC5643 \\
\hline & Predicted protein & Unknown & TC2384 \\
\hline & ABL167Cp & Unknown & TC35322 \\
\hline & Unknow protein & Transcription regulation & TC43271 TC31296 \\
\hline & Os02g0776400 protein & Transcription regulation & TC17857 ТC48466 ТC31225 \\
\hline & NADH dehydrogenase I chain M & Metabolism & TC9084 \\
\hline & Methionine S-methyltransferase & Transcription regulation & TC15884 \\
\hline \multirow[t]{2}{*}{171} & Scl1 protein & Unknown & FL816450 TC26013 \\
\hline & Os05g0562400 protein & Transcription regulation & FL708626 \\
\hline \multirow[t]{3}{*}{$399 \mathrm{a}$} & Expressed protein & Unknown & FE627710 \\
\hline & Tetratricopeptide TPR_2 repeat protein precursor & Transcription regulation & FL689938 \\
\hline & Salt-induced AAA-Type ATPase & Signal transduction & TC26446 \\
\hline $399 b$ & Expressed protein & Unknown & FE627710 \\
\hline
\end{tabular}


(Continued)

\begin{tabular}{|c|c|c|c|}
\hline miRNA & Target protein & Target function & Target gene \\
\hline \multirow{10}{*}{$444 \mathrm{a}$} & Tetratricopeptide TPR_2 repeat protein precursor & Transcription regulation & FL689938 \\
\hline & Chromosome chr12 scaffold_47 & & FL802301 \\
\hline & $\begin{array}{l}\text { MIKC-type MADS-box transcription factor } \\
\text { WM30 }\end{array}$ & Transcription regulation & FL808850 \\
\hline & MADS box protein 2 & Transcription regulation & TC45658 \\
\hline & BHLH transcription factor PTF1 & Transcription factor & TC18118 \\
\hline & $\begin{array}{l}\text { Zinc finger (C3HC4-type RING finger) pro- } \\
\text { tein-like }\end{array}$ & Transcription regulation & TC16101 \\
\hline & $\begin{array}{l}\text { Non-ribosomal peptide synthetase modules and } \\
\text { related proteins }\end{array}$ & & TC4649 \\
\hline & $\begin{array}{l}\text { Chromosome chr10 scaffold_179, whole genome } \\
\text { shotgun sequence }\end{array}$ & & TC27817 \\
\hline & $\begin{array}{l}\text { Chromosome chr7 scaffold_31, whole genome } \\
\text { shotgun sequence }\end{array}$ & & TC18635 \\
\hline & Os05g0470700 protein & Transcription regulation & TC41751 \\
\hline \multirow{3}{*}{$444 b$} & $\begin{array}{l}\text { MIKC-type MADS-box transcription factor } \\
\text { WM30 }\end{array}$ & Transcription regulation & FL808850 \\
\hline & Peptidyl-prolyl cis-trans isomerase & Signal transduction & TC1803 \\
\hline & VacJ-like lipoprotein precursor & Transcription regulation & DN148537 \\
\hline \multirow[t]{6}{*}{$528 \mathrm{a}$} & Os02g0671800 protein & Transcription regulation & GD041833 \\
\hline & Superoxide dismutase $[\mathrm{Cu}-\mathrm{Zn}]$ & Transcription regulation & TC8663 TC11635 TC51355 \\
\hline & Chromosome chr5 scaffold_2 & & TC24045 TC52797 ТC22262 \\
\hline & $\begin{array}{lll}\text { Glyoxalase/Bleomycin } & \text { resistance } & \text { pro- } \\
\text { tein/dioxygenase domain } & & \end{array}$ & Signal transduction & FL746289 \\
\hline & Plasma membrane ATPase 2 & Metabolism & FL751986 \\
\hline & $\begin{array}{l}\text { N.plumbaginifolia H+-translocating ATPase } \\
\text { mRNA }\end{array}$ & Metabolism & FL815815 \\
\hline \multirow[t]{2}{*}{$528 \mathrm{~b}$} & Predicted protein & Unknown & GD018502 \\
\hline & $\begin{array}{l}\text { Chromosome chr18 scaffold_1, whole genome } \\
\text { shotgun sequence }\end{array}$ & & TC49585 \\
\hline \multirow[t]{10}{*}{1439} & DNA (cytosine-5)-methyltransferase 1 & DNA processing & TC42883 \\
\hline & $\begin{array}{l}\text { Chromosome undetermined scaffold_109, whole } \\
\text { genome shotgun sequence }\end{array}$ & & FL703180 \\
\hline & Predicted protein & Unknown & TC24744 \\
\hline & $\begin{array}{l}\text { Chromosome undetermined scaffold_77, whole } \\
\text { genome shotgun sequence }\end{array}$ & & TC18576 \\
\hline & $\begin{array}{l}\text { Chromosome chr6 scaffold_15, whole genome } \\
\text { shotgun sequence }\end{array}$ & & TC26456 \\
\hline & Glycine C-acetyltransferase & Signal transduction & FE604006 \\
\hline & $\begin{array}{l}\text { FYVE finger-containing phosphoinositide ki- } \\
\text { nase-like }\end{array}$ & Signal transduction & TC34589 \\
\hline & DNA methylase N-4/N-6 domain protein & DNA processing & FL855194 \\
\hline & Vomeronasal receptor V1RI2 & Signal transduction & TC52734 \\
\hline & Os03g0351300 protein & Transcription regulation & TC1806 \\
\hline
\end{tabular}

targets the B1358B12 protein, T6H22.8.1 protein, MADSbox transcription factor, and miR1439 targets the winged helix transcription factor. All these genes are involved in RNA regulation, supporting the idea that miRNAs function in post-transcriptional regulation.

We also found that five miRNA families participate in metabolism. For example, miR166 targets genomic DNA, chromosome 5, TAC clone: K21P3 which has beta-galactosidase activity and participates in the carbohydrate metabolic process [56]; miR169 targets sulfur oxidation protein which is an electron carrier that functions in heme binding and iron ion binding processes; miR528 targets syringolide-induced protein B13-1-1; and miR444 targets the puta- tive P450 hydroxylase which is involved in oxidation reduction and functions as an electron carrier. P. miliaceum L. is widely planted in Northern China, where the natural environment is severe. It will be interesting to explore the idea that some of the candidate miRNAs may play roles in the ability of Panicum to adapt to environmental stress. One of the candidate miRNAs belonged to the miR1439 family that is involved in signal transduction.

The identified $P$. miliaceum L. miRNAs also target genes that control RNA transport and RNA processing. AT3g25150/MJL12_9 protein, the target of miR399, has nucleic acid binding activity and is thought to be involved in RNA transport. miR1439 targets the tRNA (guanine- 
$\mathrm{N}(1)$-)-methyltransferase that is associated with tRNA processing and modification, supporting previous reports that small RNAs are involved in mediating methylation in plants.

\subsection{Northern blot analysis}

Eight of the 12 selected miRNAs had positive signals with lengths around $21 \mathrm{nt}$. The miRNAs from three of the families, miR399, miR444, miR1439 failed the verification (Figure 4).

The miR399 family has been reported to target a putative ubiquitin conjugating enzyme (UBC), and miR399 is highly induced in low-phosphate stress conditions [57,58]. This may explain why we did not see a positive signal for miR399 under normal growth conditions. However, miR399 may contribute to the high resistance to stress of Panicum.

miR444 targets a MAD box transcription factor that is expressed during inflorescence and fruit development. Therefore, it is possible that miR444 is highly expressed only in the reproductive growth stage [59] and this may be the reason for the high yield of Panicum in arid regions.

The function of miR1439 is still not clear; however, high-throughput sequencing carried out by another group indicated that miR1439 might be related to salt-tolerance in rice [60]. Although miR1439 was found at very low frequency (2/80990) in a salt stress library and was not present in the control library, it did have a positive signal in a Northern blot assay in both the salt stress and control libraries [60]. However, perhaps because of the normal growth conditions that we used and/or the species variation, miR1439 was not detected in our Northern blot assay.

The negative controls (probes 5S rRNA, EST1, and
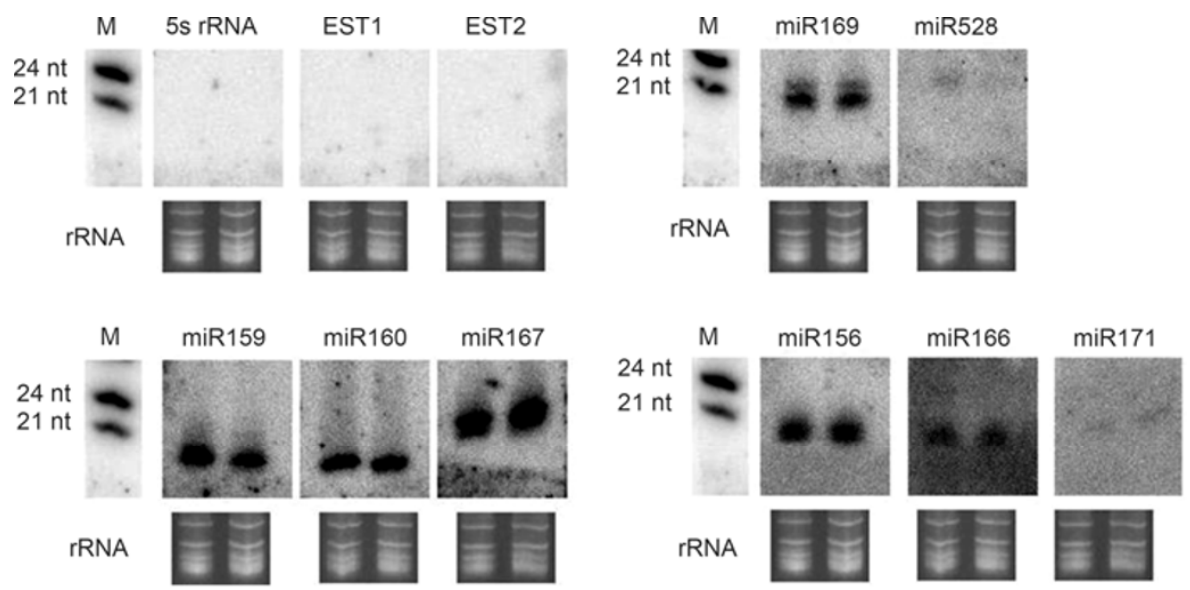

Figure 4 Northern blot hybridization assays. M, RNA marker with 3'-methyl group. rRNA, total RNA sample volume in the different lanes. The name of the miRNA is at the top of each panel. The left lane of each membrane is the Longmi-5 cultivar, the right lane is the Ningmi-9 cultivar. 5s rRNA, EST1, and EST2 are the controls that were used to monitor the effect caused by RNA degradation. No signals were detected from the controls, indicating that degradation had little effect on the results. The miR156, miR159, miR160, miR166, miR167, miR169, miR171, and miR528 candidate miRNAs produced hybrid signals that were located around $21 \mathrm{nt}$. No detectable signals were seen for miR399, miR444a, miR444b, or miR1439. 
A. Pre-miR 156a

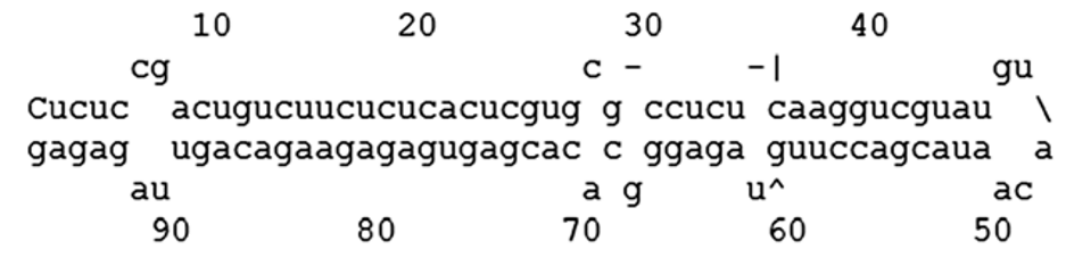

B. Pre-miR 528a

\begin{tabular}{|c|c|c|c|c|}
\hline & 10 & 20 & 30 & 40 \\
\hline a & $a$ & ggag & & ucuc au \\
\hline yag & guag gggagga & & acguacggcgaagcu & gc u \\
\hline uc & cguc cccuccu & & ugcgugccgcuucga & \\
\hline & $80^{g}$ & $9---$ & 60 & $\operatorname{uucc}_{50} \mathrm{cu}$ \\
\hline
\end{tabular}

Figure 5 Representative secondary structures of the Panicum candidate miRNAs. A, Stem-loop structure of miR156a-1. B, Stem-loop structure of miR528a-3. The actual lengths of the pre-miRNAs may be slightly shorter or longer than shown.

This work was supported by the National Natural Science Foundation of China (Grant Nos. 30600384 and 50809068), and the Young Outstanding Scholar Foundation of Northwest A \& F University, China. We thank Wang QinHu for insightful suggestions on some particulars of the study. We are grateful to Professor Hu YinGang for providing the Panicum seeds. We also thank Deng XiLian for access to the high performance computers in the Network Information Center, Northwest A \& F University, China.

1 Murchison E P, Hannon G J. MiRNAs on the move: miRNA biogenesis and the RNAi machinery. Curr Opin Cell Biol, 2004, 16: 223-229

2 Lee R C, Feinbaum R L, Ambros V. The C. elegans heterochronic gene lin-4 encodes small RNAs with antisense complementarity to lin-14. Cell, 1993, 75: 843-854

3 Lagos-Quintana M, Rauhut R, Lendeckel W, et al. Identification of novel genes coding for small expressed RNAs. Science, 2001, 294: 853-858

4 Khvorova A, Reynolds A, Jayasena S D. Functional siRNAs and miRNAs exhibit strand bias. Cell, 2007, 131: 41-49

5 Carrington J C, Ambros V. Role of microRNAs in plant and animal development. Science, 2003, 301: 336-338

6 Zhang B H, Wang Q L, Wang K B, et al. Identification of cotton microRNAs and their targets. Gene, 2007, 397: 26-37

7 Zhang B H, Pan X P, Anderson T A. MicroRNA: a new player in stem cells. J Cell Physiol, 2006, 209: 266-269

8 Zhang B H, Pan X P, Cobb G P, et al. Plant microRNA: a small regulatory molecule with big impact. Dev Biol, 2006, 289: 3-16

9 Jones-Rhoades M W, Bartel D P. Computational identification of plant microRNAs and their targets, including a stress-induced miRNA. Mol Cell, 2004, 14: 787-799

10 Saini H K, Griffiths-Jones S, Enright A J. Genomic analysis of human microRNA transcripts. Proc Natl Acad Sci USA, 2007, 104: 17719-17724

11 Fujita S, Iba H. Putative promoter regions of miRNA genes involved in evolutionarily conserved regulatory systems among vertebrates. Bioinformatics, 2008, 24: 303-308

12 Zuloaga F O, Giussani L M, Morrone O. On the taxonomic position of Panicum aristellum (Poaceae: Panicoideae: Paniceae). Syst Bot, 2006, 31: 497-505

13 Peiris H, Ibrahim M N M. Nutritive value of guinea grass (Panicum maximum Jacq.) and urea supplemented rice straw for cattle. Asian
Austral J Anim Sciences, 1995, 8: 83-88

14 Llave C, Kasschau K D, Rector M A, et al. Endogenous and silencing-associated small RNAs in plants. Plant Cell, 2002, 14: 1605-1619

15 Grad Y, Aach J, Hayes G D, et al. Computational and experimental identification of $C$. elegans microRNAs. Mol Cell, 2003, 11: 1253-1263

16 Lau N C, Lim L P, Weinstein E G, et al. An abundant class of tiny RNAs with probable regulatory roles in Caenorhabditis elegans. Science, 2001, 294: 858-862

17 Lee R C, Ambros V. An extensive class of small RNAs in Caenorhabditis elegans. Science, 2001, 294: 862-864

18 Mourelatos Z, Dostie J, Paushkin S, et al. miRNPs: a novel class of ribonucleoproteins containing numerous microRNAs. Gene Dev, 2002, 16: 720-728

19 Park W, Li J J, Song R T, et al. CARPEL factory, a Dicer homolog, and HEN1, a novel protein, act in microRNA metabolism in Arabidopsis thaliana. Curr Biol, 2002, 12: 1484-1495

20 Reinhart B J, Weinstein E G, Rhoades M W, et al. MicroRNAs in plants. Gene Dev, 2002, 16: 1616-1626

21 Yoon S, De Micheli G. Computational identification of microRNAs and their targets. Birth Defects Res, 2006, 78: 118-128

22 Wang X J, Reyes J L, Chua N H, et al. Prediction and identification of Arabidopsis thaliana microRNAs and their mRNA targets. Genome Biol, 2004, 5: R65

23 Adai A, Johnson C, Mlotshwa S, et al. Computational prediction of miRNAs in Arabidopsis thaliana. Genome Res, 2005, 15: 78-91

24 Zhang B H, Pan X P, Wang Q L, et al. Identification and characterization of new plant microRNAs using EST analysis. Cell Res, 2005, 15: 336-360

25 Lai E C, Tomancak P, Williams R W, et al. Computational identification of Drosophila microRNA genes. Genome Biol, 2003, 4: R42

26 Sunkar R, Jagadeeswaran G. In silico identification of conserved microRNAs in large number of diverse plant species. BMC Plant Biol, 2008, 8: 37

27 Altinkut-Uncuoglu A, Ercan S, Ertugrul F, et al. An EST-SSR marker linked with yellow rust resistance in wheat. Biol Plantarum, 2010, 54: 691-696

28 Cupertino F B, Leal J B, Correa R X, et al. Genetic diversity of Eucalyptus hybrids estimated by genomic and EST microsatellite markers. Biol Plantarum, 2011, 55: 379-382

29 Zheng T G, Qiu W M, Fan G E, et al. Construction and characterization of a cDNA library from floral organs and fruitlets of Citrus reticulata. Biol Plantarum, 2011, 55: 431-436 
30 Rajewsky N, Socci N D. Computational identification of microRNA targets. Dev Biol, 2004, 267: 529-535

31 Arteaga-Vazquez M, Caballero-Perez J, Vielle-Calzada J P. A family of microRNAs present in plants and animals. Plant Cell, 2006, 18: 3355-3369

32 Altschul S F, Gish W, Miller W, et al. Basic local alignment search tool. J Mol Biol, 1990, 215: 403-410

33 Zhang B, Pan X, Cannon $\mathrm{C} \mathrm{H}$, et al. Conservation and divergence of plant microRNA genes. Plant J, 2006, 46: 243-259

34 Dezulian T, Remmert M, Palatnik J F, et al. Identification of plant microRNA homologs. Bioinformatics, 2006, 22: 359-360

35 Zhu J K. Reconstituting plant miRNA biogenesis. Proc Natl Acad Sci USA, 2008, 105: 9851-9852

36 Steffen P, Voss B, Rehmsmeier M, et al. RNAshapes: an integrated RNA analysis package based on abstract shapes. Bioinformatics, 2006, 22: 500-503

37 Reeder J, Giegerich R. Consensus shapes: an alternative to the Sankoff algorithm for RNA consensus structure prediction. Bioinformatics, 2005, 21: 3516-3523

38 Giegerich R, Voss B,Rehmsmeier M. Abstract shapes of RNA. Nucleic Acids Res, 2004, 32: 4843-4851

39 Zhang B H, Pan X P, Cox S B, et al. Evidence that miRNAs are different from other RNAs. Cell Mol Life Sci, 2006, 63: 246-254

40 Dai X B, Zhao P X. psRNATarget: a plant small RNA target analysis server. Nucleic Acids Res, 2011, 39: W155-159

41 Hornstein E, Mansfield J H, Yekta S, et al. The microRNA miR-196 acts upstream of Hoxb8 and Shh in limb development. Nature, 2005, 438: 671-674

42 Yamada-Akiyama H, Akiyama Y, Ebina M, et al. Analysis of expressed sequence tags in apomictic guineagrass (Panicum maximum). J Plant Physiol, 2009, 166: 750-761

43 Cerda J, Mercade J, Lozano J J, et al. Genomic resources for a commercial flatfish, the Senegalese sole (Solea senegalensis): EST sequencing, oligo microarray design, and development of the bioinformatic platform Soleamold. BMC Genomics, 2008, 9: 508

44 Kuster H, Becker A, Firnhaber C, et al. Development of bioinformatic tools to support EST-sequencing, in silico- and microarray-based transcriptome profiling in mycorrhizal symbioses. Phytochemistry, 2007, 68: 19-32

45 Li L, Brunk B P, Kissinger J C, et al. Gene discovery in the apicomplexa as revealed by EST sequencing and assembly of a comparative gene database. Genome Res, 2003, 13: 443-454
46 Paquola A C, Nishyiama M Y Jr., Reis E M, et al. ESTWeb: bioinformatics services for EST sequencing projects. Bioinformatics, 2003, 19: 1587-1588

47 Wang J P, Lindsay B G, Cui L, et al. Gene capture prediction and overlap estimation in EST sequencing from one or multiple libraries. BMC Bioinformatics, 2005, 13: 300

48 Rhoades M W, Reinhart B J, Lim L P, et al. Prediction of plant microRNA targets. Cell, 2002, 110: 513-520

49 Cardon G H, Hohmann S, Nettesheim K, et al. Functional analysis of the Arabidopsis thaliana SBP-box gene SPL3: a novel gene involved in the floral transition. Plant J, 1997, 12: 367-737

50 Cardon G, Hohmann S, Klein J, et al. Molecular characterisation of the Arabidopsis SBP-box genes. Gene, 1999, 237: 91-104

51 Kranz H D, Denekamp M, Greco R, et al. Towards functional characterisation of the members of the R2R3-MYB gene family from Arabidopsis thaliana. Plant J, 1998, 16: 263-276

52 Stracke R, Werber M,Weisshaar B. The R2R3-MYB gene family in Arabidopsis thaliana. Curr Opin Plant Biol, 2001, 4: 447-456

53 Theologis A, Ecker J R, Palm C J, et al. Sequence and analysis of chromosome 1 of the plant Arabidopsis thaliana. Nature, 2000, 408: 816-820

54 Yamada K, Lim J, Dale J M, et al. Empirical analysis of transcriptional activity in the Arabidopsis genome. Science, 2003, 302: 842-846

55 Green K A, Prigge M J, Katzman R B, et al. CORONA, a member of the class III homeodomain leucine zipper gene family in Arabidopsis, regulates stem cell specification and organogenesis. Plant Cell, 2005, 17: 691-704

56 Asamizu E, Sato S, Kaneko T, et al. Structural analysis of Arabidopsis thaliana chromosome 5. VIII. Sequence features of the regions of 1081958 bp covered by seventeen physically assigned P1 and TAC clones. DNA Res, 1998, 5: 379-391

57 Sunkar R, Zhu J K. Novel and stress-regulated microRNAs and other small RNAs from Arabidopsis. Plant Cell, 2004, 16: 2001-2019

58 Fujii $\mathrm{H}$, Chiou $\mathrm{T} \mathrm{J}$, Lin $\mathrm{S} \mathrm{I}$, et al. A miRNA involved in phosphate-starvation response in Arabidopsis. Curr Biol, 2005, 15: 2038-2043

59 Sunkar R, Girke T, Jain P K, et al. Cloning and characterization of microRNAs from rice. Plant Cell, 2005, 17: 1397-1411

60 Sunkar R, Zhou X, Zheng Y, et al. Identification of novel and candidate miRNAs in rice by high throughput sequencing. BMC Plant Biol, 2008, 8: 25

Open Access This article is distributed under the terms of the Creative Commons Attribution License which permits any use, distribution, and reproduction in any medium, provided the original author(s) and source are credited. 